\title{
Sprachwechsel in der neuesten litauischen Migrations- und Mobilitätsliteratur
}

\author{
RŪTA EIDUKEVIČIENĖ
}

\begin{abstract}
Language Change in the Most Recent Lithuanian Literature of Migration and Mobility. When discussing literary multilingualism within the Lithuanian literary scene, researchers usually refer to different groups of authors. Some were born and socialised abroad immediately after the Second World War, some left Lithuania after 1990, but producing their texts in different linguistic contexts all of them write consistently in one language, English or Lithuanian. In the most recent Lithuanian migration and mobility literature, however, one can observe examples of intra-textual bilingualism or multilingualism, which illustrate the integration problems of Lithuanian (labour) migrants into foreign societies on the one hand and the development of multiple global identities on the other. The paper examines these spreading tendencies focusing on the exemplary novel Stasys Šaltoka (2017) by Gabija Grušaite and discussing the structure and functions of the intra-textual code-switching. The creative use of language directed against conventional linguistic purism shows that the young generation of Lithuanian authors tends to break language and cultural borders. The authors Unè Kaunaitè, Gabija Grušaite and others show that the playful use of multilingualism can be subversive, ironic, but at the same time can highlight the problems of language dominance and thus political, social and cultural exclusion, express group mentality, identity changes, etc. The way that Lithuanian literature deals with multilingualism, expressed explicitly or implicitly, reveals the extent to which certain literary texts can be described as transcultural, in line with the current tone of European and global migration and mobility literature.
\end{abstract}

Keywords: migration; mobility literature; code-switching; Lithuania; Gabija Grušaitè

\section{Einleitung}

Im Jahr 2017 fand die Vilniusser Buchmesse unter dem Motto „Litauische Zeichen in der Welt" statt, womit die Veranstalter den Versuch unternommen haben, das Lesepublikum mit den weltweit zerstreuten Spuren litauischer Literatur vertraut zu machen. Besondere Aufmerksamkeit galt dabei jenen 
Autoren litauischer Abstammung, die unmittelbar nach dem Zweiten Weltkrieg in den DP-Lagern, den USA oder Kanada geboren und dort sozialisiert wurden, sich häufig litauischer Themen und Motive bedienen, aber ihre Texte auf Englisch und in erster Linie für ein englischsprachiges Lesepublikum schreiben, wie z.B. Birutė Putrius, Antanas Šileika, Irena Mačiulytė-Guilford. Die Autorengeneration, die Litauen um bzw. nach 1990 verlassen hat, z.B. die seit 1989 auf Zypern ansässige Autorin Dalia Staponkutė oder der in Frankreich lebende Nationalpreisträger Valdas Papievis, schreiben hingegen ausschließlich in litauischer Sprache, auch wenn sie in ihren Texten Themen wie Migration, Identität oder Transkulturalität behandeln. Staponkutè, die den Themenkomplex Sprachreflexion, Sprachkritik, Zweisprachigkeit und Mehrsprachigkeit konsequent diskutiert, behauptet, „die Sprache sei ein Verhandlungsraum“, sie selbst sei „ein gespaltener Mensch und Autorin“ und „das Sprechen über die Sprache sei die einzige Möglichkeit, diese Spaltung zu überwinden", aber sie sei skeptisch, ob das Schreiben in einer Fremdsprache für sie als Autorin in Frage käme (Interview am 23.2.2017).

Gerade in Hinblick darauf, dass sich in der neuen litauischen Migrationsund Mobilitätsliteratur ${ }^{1}$ keine Sprachgrenzüberschreitungen verzeichnen lassen, die anderen Sprachen die Identität der Autoren und Autorinnen nicht entscheidend durchdringen und „die Möglichkeit, in einer anderen Sprache als der Erstsprache zu schreiben, nur theoretisch existiert“, stellt die Literaturwissenschaftlerin Dalia Satkauskytè die berechtigte Frage, ob sich für die Analyse dieser Literatur theoretische Ansätze hybrider kultureller Identitäten, so wie sie von Homi K. Bhabha oder Gayatri Spivak formuliert wurden, überhaupt eignen, obwohl sie in der litauischen Forschung am häufigsten Anwendung finden (Satkauskytè 2011: 122). Eine ähnliche Position vertritt Eglè Kačkutè, die ebenfalls das Fehlen der Belege für sprachliche Hybridität in der litauischen Literatur konstatiert und dazu rät, in dem Fall nicht von sprachlich und kulturell hybriden Identitäten, sondern nur von einzelnen „Erscheinungsformen der Hybridität“ zu sprechen (Kačkutè 2013).

Nach dem EU-Beitritt (2004) hat sich Litauen zu einer der mobilsten Nationen in der EU entwickelt (Eurostat-Bericht 06/2020), was auch einen enormen Aufschwung der neuen Migrations- und Mobilitätsliteratur zur Folge hatte. Diese Literatur ist in den letzten Jahren zu einem populären

Der erweiterte Begriff „Mobilitätsliteratur“ ist der Studie Transcultural Writers and Novels in the Age of Global Mobility (2015) von Arianna Dagnino entliehen: „literatures of mobility [...] include those works of fiction which are affected and shaped by migration flows, wanderlust, travel experiences, diasporic, exile, or postcolonial conditions, expatriate statuses, and, more recently, the multiple trajectories of transnational and neonomadic movements" (Dagnino 2015: 145). 
Forschungsgegenstand litauischer Literaturwissenschaft geworden (Beiträge von Žydronè Kolevinskienè, Dalia Kuizinienè, Laura Laurušaitè, etc.), aber aus den oben erwähnten Gründen wird sie kaum unter dem Aspekt der Zweisprachigkeit bzw. Mehrsprachigkeit, sei es auf der Autoren- oder Textebene, diskutiert. Zu erwähnen ist an dieser Stelle der Aufsatz von Laura Laurušaitè Kalbos transformacinè galia lietuvių ir latviu (e)migracijos prozoje (Die Transformationskraft der Sprache in der litauischen und lettischen (E)Migrationsprosa, 2019), der sich in Anlehnung an postkoloniale Theorieansätze mit den Einstellungen der Migranten zur Erstsprache und Fremdsprache sowie sprachlich motivierten Diskriminierungs- bzw. Dominanzverhältnissen in der jüngsten litauischen und lettischen Migrationsliteratur beschäftigt. Laurušaitè diskutiert nicht nur die fehlende Fremdsprachenkompetenz und dadurch bedingte Handlungsunfähigkeit der baltischen (Arbeits-)Migranten, sondern auch die zusammen mit der Erlangung neuer Sprachkenntnisse einhergehende Transformation ihrer kulturellen Identität sowie ihre (angebliche) Entfremdung von der Erstsprache, die für die Hauptursache kultureller Ortslosigkeit erklärt wird (als Beispiele werden Texte von Paulina Pukytè, Audronè Urbonaitė, Unė Kaunaitè u.a. angeführt). Es ist der Forscherin zuzustimmen, dass in den Migrationskontexten der Sprache eine viel größere Bedeutung denn als bloßes Kommunikationsmittel zukommt. Etwas verkürzt werden jedoch in dem Aufsatz die komplexen Sprachkontakte auf Konfliktsituationen bzw. paradoxe lapsus linguae reduziert (Laurušaitė 2019: 40ff) und die baltischen (Arbeits-)Migranten in die Rolle der Sprachlosen hineinversetzt, die sich nur innerhalb ihres Sprachkollektives bewegen oder sich in die Aufnahmegesellschaft auf Kosten ihrer Erstsprache und ihres kulturellen Selbstbewusstseins integrieren.

Während Laurušaitè die zentrale Funktion literarischer Mehrsprachigkeit in der Erzeugung von Irritation sieht, bleiben andere aktuelle Mobilitätsmodelle und literarische Entwürfe sprachlicher und kultureller Identität außer Acht. Beachtenswert ist in diesem Zusammenhang der Erfolgsroman Stasys Šaltoka von Gabija Grušaitè (2017), der ein neuartiges flexibles Selbstverständnis eines Litauers mit Migrationshintergrund präsentiert und einen freien bzw. kreativen Umgang mit der Mehrsprachigkeit illustriert. Der Romanprotagonist, ein New Yorker Hipster und aktiver Nutzer von Social Media, präsentiert den Typus eines Globetrotters, der verschiedene Sprachen spricht, darüber aber kaum reflektiert, denn Zweisprachigkeit bzw. Mehrsprachigkeit scheint für ihn eine Selbstverständlichkeit zu sein. Grušaitè liefert einen Beleg dafür, dass es auch solche litauischen Auswanderer gibt, die innere und äußere Sprachkonflikte überwunden zu haben scheinen, quersprachige Kompetenz aufweisen und gerne Sprachkonventionen brechen 
(Kačkutè 2018). So werden in den litauischen Text, gegen jegliche Normen des Sprachpurismus verstoßend, englischsprachige Elemente integriert, ohne diese speziell zu markieren oder zu kommentieren, sodass sowohl der Ich-Erzähler als auch das Buch selbst als explizit zweisprachig bezeichnet werden können.

Eben diesem neuen Typus sprachlicher Hybridität sowie dem textästhetischen Verfahren des Sprachwechsels in der neuesten litauischen Migrationsund Mobilitätsliteratur soll in dem vorliegenden Beitrag die größte Aufmerksamkeit gelten. Die Fragestellung ist unter anderem durch die Bemerkung Till Dembecks motiviert, dass „eine Darstellung von Sprachwechsel und -mischung in postkolonialen und postmigratorischen Texten [...] über die Grenzen der (west-)europäischen und amerikanischen Literatur hinausgehen“ müsste (Dembeck 2020: 144). Wie von Dembeck bemerkt, wird die Interpretation des Sprachwechsels „sowohl die allgemeine soziokulturelle Wertigkeit der verwendeten Sprachen als auch die vom Text selbst erzeugte Sprach- und Kommunikationssituation" (ebd. 146) genau in den Blick nehmen und die Funktionen eines derartigen Verfahrens am Beispiel eines Romans diskutieren.

\section{Theoretische und methodische Grundlagen}

Für die Analyse des Sprachwechsels bieten zwei Studien eine haltbare methodische Grundlage, nämlich Gulia Radaellis Literarische Mehrsprachigkeit (2011) und Werner Helmichs Ästhetik der Mehrsprachigkeit (2016). Beide Forscher haben ausführliche Beschreibungsmodelle für mehrsprachige literarische Texte entwickelt, obwohl sie ein unterschiedliches Verständnis des Phänomens haben und voneinander abweichende Herangehensweisen an die konkreten Texte vorschlagen. Beiden ist aber gemeinsam, dass sie den Begriff Mehrsprachigkeit nicht auf das Nebeneinander verschiedensprachiger Texte innerhalb einer Nationalliteratur, auch nicht auf das Nebeneinander verschiedensprachiger Texte im Werk eines Autors oder einer Autorin, sondern auf die innertextuelle Mehrsprachigkeit beziehen (Radaelli 2011: 48; Helmich 2016: 14). Wenn es um das Vorkommen anderssprachiger Elemente innerhalb eines Textes geht, sind Sprachwechsel und Sprachmischung die entscheidenden Phänomene, die sich aber nicht scharf, eher nach der Art der Betrachtung (quantitativ oder qualitativ) voneinander unterscheiden lassen (Helmich 2016: 18). Auch in dem vorliegenden Beitrag werden die Begriffe parallel benutzt, wobei grundsätzlich die von Dembeck vorgeschlagene Definition gilt: „Als Sprachwechsel ist zu bezeichnen, wenn in einem Text Segmente, die unterschiedlichen Idiomen zuzuordnen sind, aufeinander folgen, wohingegen man von Sprachmischung 
bei solchen Texten oder Textteilen sprechen kann, in denen zwei Idiome zu unterscheiden sind, ohne dass sie sich einzelnen Segmenten zuordnen ließen“ (Dembeck 2020: 125).

Der wichtigste Unterschied zwischen Radaelli und Helmich besteht in der Wahrnehmbarkeit und der Auffassung der in einem Text enthaltenen Sprachen, was nicht zuletzt mit ihrem Untersuchungsgegenstand zusammenhängt. Helmich versucht, einen umfangreichen Textkorpus zu bearbeiten, weshalb er sich alleine schon aus pragmatischen Gründen dafür entscheidet, von Sprachwechsel nur dann zu sprechen, wenn die involvierten Sprachen im Text manifest sind (Helmich 2016: 17). Radaelli geht von einem viel breiteren Begriff des Sprachwechsels bzw. der Sprachmischung aus. Zur systematischen Einteilung und deskriptiven Erfassung der Mehrsprachigkeit literarischer Texte schlägt sie drei heuristische Grundkriterien vor: der Fokus (mehrsprachiges Einzelwerk oder mehrsprachiges Gesamtwerk eines Autors), die Wahrnehmbarkeit (manifeste oder latente Mehrsprachigkeit) und die Sprachen (Einzelsprachen bzw. Nationalsprachen, Sprachvarietäten oder erfundene Sprachen) (Radaelli 2011: 47).

Für die Analyse manifester Formen des Sprachwechsels bzw. der Sprachmischung in einem konkreten Text bieten sich die von Helmich übersichtlich zusammengestellten Kriterien (Helmich 2016: 30ff) an, die später auch bei der Erläuterung der fremdsprachigen Elemente im Roman Stasys Šaltoka zu Hilfe gezogen werden: 1) Markierung bzw. graphische Hervorhebung; 2) Lokalisierung im Werk; 3) Einzelgröße und Gesamtumfang; 4) Durchdringungs- oder Vermischungsgrad; 5) Figuren- und Erzählerrede; 6) Verständnishilfen; 7) Konnotationen einzelner Fremdsprachen; 8) Korrektheitsgrad. Im Unterschied zu Helmich berücksichtigt Radaelli auch die latente Mehrsprachigkeit, bei der „andere Sprachen nur unterschwellig vorhanden und nicht unmittelbar wahrnehmbar sind“, und betrachtet diese als die häufigste Form von literarischer Mehrsprachigkeit überhaupt (Radaelli 2011: 61). Zu solchen latenten Formen zählen die Übersetzung, die Sprachreflexion sowie die im Text auftretenden Sprachverweise (ebd. 61f). Darüber hinaus können literarische Texte auf eine andere Sprache oder auf Mehrsprachigkeit Bezug nehmen, indem sie mehrsprachige Begegnungen und Räume schildern. Aufenthalte im Ausland, Reise oder Auswanderung stellen laut Radaelli wichtige Topoi mehrsprachiger Literatur dar (ebd. 65).

Neben den besonders sichtbaren Fällen, bei denen der Sprachwechsel zwischen zwei oder mehreren Einzelsprachen stattfindet, sollten im Sinne des Beschreibungsmodells von Radaelli auch sprachliche Varietäten wie Dialekte, Idiolekte, Soziolekte, etc. beachtet werden (Radaelli 2011: 70). Dabei solle auch gefragt werden, welche Bedeutung den jeweiligen Einzelsprachen oder 
Varietäten im mehrsprachigen Text zukommt, so etwa wenn eine Sprache an einen bestimmten topischen bzw. stereotypen Inhalt gekoppelt ist und der Darstellung von National- oder Berufscharakteren dienen soll (ebd.).

An dieser Stelle soll etwas näher auf die Aufsätze hingewiesen werden, die sich mit Funktionen der Mehrsprachigkeit in literarischen Texten auseinandersetzen. In der neueren Forschung ist meistens von „erfahrener“ und „ästhetischer“ Funktion der Mehrsprachigkeit die Rede ist: Man unterscheidet „zwischen solchen Formen literarischer Mehrsprachigkeit, die ihren Dreh- und Angelpunkt im Leben selbst, sprich in der mehrsprachigen Erfahrung je unterschiedlicher Gruppen haben; und solchen Formen, die in erster Linie aus einem ästhetischen Willen heraus erklärt werden können“ (Dembeck/Uhrmacher 2016: 10). Dembeck und Uhrmacher kritisieren diese Unterscheidung, insbesondere wenn einer von diesen Formen eine Priorität eingeräumt wird, und raten - was aus unserer Perspektive viel Sinn macht - dazu, beide Aspekte in den Blick zu nehmen:

\begin{abstract}
Denn zum einen besteht der Verdacht, dass Ansätze, die allein dem Populären eine kollektive und lebensunmittelbare Herkunft unterstellen, dabei die Fähigkeiten literarischer Werke unterschätzen. Diese können wirkmächtig alternative Weltentwürfe ins Spiel bringen und dürfen nicht als individualistisches Glasperlenspiel abgewertet werden. Zum anderen ist klar, dass kein literarisches Werk seinen Umgang mit Sprachdifferenzen im luftleeren Raum pflegt. Im Gegenteil: Ihre kulturelle und soziale Wertigkeit ist unmittelbar Teil jeder ästhetischen Konstruktion von Sprachdifferenzen. (Ebd. 11)
\end{abstract}

Diese Bemerkung soll insbesondere für die Beschäftigung mit der mehrsprachigen Migrations- und Mobilitätsliteratur gelten, denn bei solchen Texten, die sich mit verschiedenen Formen sozialer Integration bzw. Exklusion beschäftigen, ist die Neigung groß, das sprachliche Leben unserer Gesellschaften den individuellen ,ästhetischen Spielen“ vorzuziehen.

Ohne die gewonnene Erkenntnis, dass mehrsprachige Texte sowohl hinsichtlich ihrer gesellschaftlicher Relevanz als auch ihrer ästhetischen Gestaltung zu bewerten sind, ganz aus den Augen zu verlieren, soll hier ein kurzer Blick auf die Studie Global Playing in der Literatur von Elke Sturm-Trigonakis (2007) geworfen werden, in der die Forscherin das transnationale, kosmopolitische Bewusstsein mehrsprachiger Werke als Reflex der Globalisierungsthematik in den Werken selbst interpretiert und es in ihre Definition von „Neuer Weltliteratur“ mit hineinnimmt. Für die „Neue Weltliteratur“ sollen Sturm-Trigonakis zufolge solche Merkmale gelten, wie eine weit definierte, auf Sprachvarietäten wie Soziolekte und Dialekte ausgedehnte Mehrsprachigkeit der Texte, die „Verarbeitung eines wie auch immer gearteten Globalisierungsdiskurses“, aber auch die 
Sprachwechsel in der neuesten litauischen Migrations- und Mobilitätsliteratur

„Hinwendung zum Regionalen und Lokalen“ (Sturm-Trigonakis 2007: 20, 109). Die Forscherin bemerkt, dass in der von ihr untersuchten Literatur sowohl die Menge als auch die Komplexität der Texte, die Sprachwechsel und Sprachmischung als Verfahren benutzen, stark gestiegen ist (ebd. 160-163). Dembeck weist auch darauf hin, dass die Texte, die sich mit Migration oder mit einem (post-)kolonialen Kontext in Verbindung bringen lassen, mit Blick auf Sprachwechsel und Sprachmischung derzeit das größte Interesse von Seiten der Forschung erhalten (Dembeck 2020: 143). Aus literaturwissenschaftlicher Perspektive gelte es dabei, „sich mit der Frage auseinanderzusetzen, in welchen einzelnen literarischen Texten sich unter welchen Rahmenbedingungen und wann welche Formen von Sprachwechsel und/oder Sprachmischung finden, welche kulturpolitische Relevanz sie haben und wie sie in einen historischen Zusammenhang gebracht werden können“ (ebd. 127). Eben um eine ausführliche Erläuterung von verschiedenen Manifestationen des Sprachwechsels bzw. der Sprachmischung und ihren in einem konkreten Text angelegten gesellschaftlich relevanten, aber auch ästhetischen Funktionen soll es in einem weiteren Schritt gehen.

\section{Sprachliche und kulturelle Hybridität in Gabija Grušaitės Roman Stasys Šaltoka}

Gabija Grušaitè, die Autorin des Romans Stasys Šaltoka, wurde 1987 in Vilnius geboren, sie studierte Soziale Medien und Anthropologie in London, lebte sechs Jahre in Malaysien, gründete dort das Kunstzentrum „Hin Bus Depot“ und reiste viel. Der Roman Stasys Šaltoka wurde 2018 mit dem renommierten Jurga Ivanauskaité-Literaturpreis ausgezeichnet und in demselben Jahr unter dem Titel Cold East ins Englische übersetzt, 2019 erhielt die Autorin den Penang Monthly Literaturpreis. Als der Roman 2018 auf dem Festival für Neue Europäische Literatur in New York vorgestellt wurde, bemerkte die Literaturkritikerin Violeta Kellertas, dass dieser Roman die litauische Literatur „bombenartig“ ins 21. Jahrhundert führt (vgl. die Rückseite des Schutzumschlags).

Es gehört dazu, dass die globale Instagram-Generation des 21. Jahrhunderts, von der im Roman die Rede ist, in ihrer Kommunikation immer wieder Sprach- und Kulturgrenzen überschreitet, was von einigen litauischen Kritikern als Normverstoß verstanden wurde:

Die Meinungen gehen hierzu weit auseinander: Kritisiert (aber auch positiv wahrgenommen) wurde der umgangssprachliche Ton, zu dem natürlich auch Anglizismen gehören, also all das, was die älteren Sprachredakteure als 
syntaktische Fehler, unzulässigen Jargon oder Schimpfwörter wegzustreichen pflegten. Kritisiert wurde auch die im Roman beschriebene, von einem Teil dieser Generation gewählte Lebensweise. Der Romanprotagonist und seine Freunde reden so wie viele ihrer Zeitgenossen, die die Welt kreuz und quer bereist haben, aber immer noch hier sind, gespalten zwischen Kulturen, Religionen und Lebensweisen: drei englische Wörter, zwei litauische und darauffolgend ein russisches Schimpfwort. (Kaniavienè 2018) ${ }^{2}$

Laura Laurušaitė betrachtet die im Roman vorkommende Mehrsprachigkeit als „Zeichen der Primitivität des sprachlichen Ausdrucks", als Hinweis auf die „Wurzellosigkeit“ der heutigen globalen Generation und die „Demythologisierung der Muttersprache“; sie konstatiert eine vermehrte Anzahl solcher fiktiven „Sprachgrenzüberschreitungen“ in der neuesten litauischen und lettischen Literatur und zieht das Fazit, dass die Sprache in diesen Texten ihre identitätsstiftende Funktion zu verlieren scheint (Laurušaitè 2019: 45).

Der Roman Stasys Šaltoka ist eine Art „Nachdichtung“, die der globalen Instagram-Generation eine authentische Stimme verleihen soll. Im Hinblick auf die Authentizität der Sprache sagt die Autorin in einem Interview:

Die „Richtigkeit“ der Sprache ist uns aufgezwungen. Dies spiegelt nicht unsere wirkliche Sprache wider, geschweige denn unser Sprechen. Das ist ein Verstoß gegen die Demokratie. Ich wollte ein Buch schreiben, das von hier und jetzt handelt. Die Sprache ist historisch. Ich denke, in 5 oder 10 Jahren werden wir anders sprechen. Ich wollte eine Art anthropologischen Text verfassen, der die heutige Sprache abbildet. Im Rückblick werden wir sagen: „Im Jahr 2017 haben die Menschen so geredet, wie lustig." Die Sprache verändert sich, sie ist nicht statisch. Man soll sie nicht reinigen, man muss sie lassen, so wie sie ist. Wenn wir denken, dass alles, was gegen die Norm verstößt, in Anführungszeichen stehen muss, wird unsere Sprache hölzern. (Grušaitè im Interview mit Karolis Vyšniauskas im Jahr 2018)

Dies sind Äußerungen, die als eine Autorenpoetik gelten können und die sich auf zentrale Anliegen der inhaltlichen und formalen Textgestaltung in Stasys Šaltoka beziehen.

Im Zentrum des Romans steht ein 29-jähriger Litauer namens Stasys Šaltoka, der Ich-Erzähler und Protagonist, der (sowie die Romanautorin selbst) zunächst in London studiert und Gelegenheitsjobs übernimmt, später in New York lebt und mit Freunden durch Südostasien reist. Auf den ersten Blick lässt sich der Roman der Gattung Migrationsliteratur zurechnen, aber

\footnotetext{
2 Alle Übersetzungen aus dem Litauischen ins Deutsche von mir, R.E.
} 
hier gilt es gewisse Vorsicht walten zu lassen, denn der Protagonist ist eigentlich ein Weltbürger (real und virtuell), der sich überall gleich gut bzw. schlecht fühlt. Seine Heimatstadt Vilnius ist nur ein Ort unter vielen anderen, ein Ort, der Erinnerungen hervorruft und für die postsowjetische Identität des Protagonisten steht, jedoch aktuell keine Berührungspunkte bietet. Die unüberwindbare Distanz wird im Gespräch mit einer amerikanischen Bekannten zum Ausdruck gebracht: Wenn Stasys verkündet, er möchte New York verlassen, fragt diese, wohin er denn gehen möchte: „Nach Los Angeles? Als ob LA der einzige Ort wäre, wohin man nach dem Verlassen dieser Stadt fahren könnte. - Nach Hause? Nach Vilnius? Ich schüttele den Kopf. Sie hat recht - was würde das alles bringen." $\left(S S_{\text {S }} 35\right)^{3}$

Stasys Šaltoka gehört nicht mehr zu denjenigen osteuropäischen Migranten, die für eine bestimmte Zeit ins Ausland gehen, um Geld zu verdienen, sondern er vertritt die Generation, die seit mehr als einem Jahrzehnt frei von jeglichen finanziellen Nöten irgendwo in der Welt lebt, ohne feste Bindungen und Sentiments (SŠ: 27). Ständige Bewegung, Dynamik und (Selbst-)Suche scheinen für diese Generation wichtiger $\mathrm{zu}$ sein als ein fester Wohnsitz. „Whatever" ist daher das am häufigsten vorkommende englische Wort im Roman, das sich sowohl auf das eigene Leben des Erzählers, auf die alltäglichen Krisen seiner New Yorker Bekannten, aber auch auf die globalen Naturund Humanitätskatastrophen bezieht. Diese Global Player fühlen sich überall zuhause, zugleich auch überall fremd, was sich vor allem durch Überfluss und Lebenslangeweile erklären lässt. Weder die Heimat noch die Erstsprache bieten für sie eine identitätsstiftende Grundlage, auch die Gemeinschaft mit den anderen entsteht eher per Zufall, und weniger durch ein Hineingeborenwerden in einen Raum oder ein Kollektiv, sondern eher durch einen Mausklick.

Wenn man zunächst eine Grobanalyse des Sprachwechsels im Roman Stasys Šaltoka durchführt und sich dabei des von Helmich vorgeschlagenen Instrumentariums bedient, so fällt auf, dass die anderssprachigen (englischen) Elemente unmarkiert in den litauischen Erzählfluss eingefügt und im ganzen Text lokalisiert werden. Was die Einzelgröße und den Gesamtumfang der englischen Einfügungen betrifft, benutzt die Autorin sowohl Einzellexeme als auch Wortverbindungen, vollständige Sätze und Textpassagen, die additiv oder synthetisch in den litauischen Text integriert werden und von einem hohen „Vermischungsgrad“ der Sprachen (Helmich 2016: 31) zeugen. Der

3 Bei der Übersetzung von litauischen Zitaten ins Deutsche sind sämtliche englischsprachigen Passagen in der Originalsprache belassen, mit der Hoffnung, dass dadurch der mehrsprachige Ton des Romans zumindest teilweise gewahrt bleibt. Die Seitennummern aus dem Roman werden mit der Abkürzung „SŠ“ angegeben. 
Roman handelt von einer zweisprachigen bzw. mehrsprachigen Generation und richtet sich an ein genauso mehrsprachiges Lesepublikum, deswegen findet man im Text keine Verständnishilfen für anderssprachige Elemente, d.h., das Fremdsprachige wird einfach stehen gelassen, was in dieser Poetik der Mehrsprachigkeit für programmatisch erachtet werden muss.

Dass Grušaitès Roman die Stimme einer mehrsprachigen Generation der Global Player um die dreißig möglichst authentisch nachahmen soll, beweist unter anderem die Tatsache, dass anderssprachige (englische) Elemente genauso häufig in der Erzähler- wie in der Figurenrede vorkommen. Bei einem Ich-Erzähler lässt sich aber nicht immer deutlich feststellen, ob es sich um erlebte Rede oder seine an andere Figuren gerichtete Aussagen bzw. seine Posts handelt. In der erlebten Rede kommen der Sprachwechsel bzw. die Sprachmischung meistens dann vor, wenn der Erzähler über andere Menschen und Beziehungen reflektiert: „Ungodly hour, wenn die Menschen wachwerden und an den bevorstehenden Tag denken, an to do lists, was sollte man erledigen und was weglassen, warum und wofür." (SŠ: 11) Wenn zwei anderssprachige Sätze miteinander kombiniert werden, ist der litauischsprachige Satz meistens neutral und der darauffolgende englischsprachige wirkt aussagekräftiger: „Zwei Küsse auf die Wangen. I could fucking murder you now“ (SŠ: 15) oder „Wo sind meine Geschenke und warum habe ich nichts bekommen. Give me my fucking presents“ (SŠ: 17). Mit Hilfe der erlebten Rede werden auch, so der Eindruck, Vorentwürfe für seine späteren, Lebensweisheiten vermittelnden Posts formuliert, so etwa: „Ich werde in 20 Minuten da sein. I have no dignity. No integrity." (SŠs: 21)

Was die Figurenrede im Text betrifft, werden die Gespräche des IchErzählers mit seinen amerikanischen Freunden, später auch mit dem Russen Alex oder mit Menschen in Asien (Hotel- oder Restaurantpersonal, Taxifahrer, etc.) in englischer Sprache geführt, aber im Text entweder nur in litauischer Sprache oder eben mit Hilfe der Sprachmischung wiedergegeben: „- Ich will meine Frau verlassen, - sagt er [ein amerikanischer Freund] zum Schluss. Go ahead - antworte ich. - Warum nicht.“ (SŠ: 23) Dass all die Gespräche vollständig in englischer Sprache stattfinden, wird an einigen Stellen indirekt verraten, so etwa, wenn bei einem Telefongespräch der Erzähler sich über den amerikanischen Akzent der Gesprächspartnerin aufregt: „Džanet [Janet], versuche ich möglichst höflich, - that's retarded. - Stasys... du brauchst Hilfe, - in dem Moment hasse ich ihren all american Akzent." (SŠ: 176) Auch mit dem Russen Alex scheint Stasys in englischer Sprache zu kommunizieren, wodurch ein klarer Unterschied zu der älteren Generation der Litauer, die in der Alltagskommunikation mit Russen Russisch bevorzugen, verdeutlich wird. Stasys verweist auf vieles, was ihn mit Alex verbindet, aber anstelle des 
Sprachwechsel in der neuesten litauischen Migrations- und Mobilitätsliteratur

Russischen als Kommunikationssprache tritt das Englische, auch wenn der osteuropäische Akzent der beiden sich nicht ganz überhören lässt:

Wir beide wollen allein in der neuen Stadt bleiben, in der Stadt, die wir nicht verstehen, aber der osteuropäische Akzent, gleiche Frisuren und die Liebe für Whisky reichen aus, um uns so zu fühlen, als würden wir einander seit hundert Jahren kennen. (SŠ: 63)

Es ist nicht ersichtlich, wie die vermutlich englischsprachige Kommunikation mit den auf den Reisen getroffenen Thailändern, Malaysiern, Mongolen, etc. funktioniert, denn auf der Textebene werden diese Gespräche in litauischer Sprache wiedergegeben.

Englische Begriffe werden immer dann eingefügt, wenn es um modische Produkt-, Berufs- oder Lebensstilbezeichnungen geht: „Ich vermisse gar nicht die New Yorker und ihre life coaches“ (SŠ: 176), „new age Idioten“ (SŠ: 64), etc. Geschimpft wird im Roman auch meistens auf Englisch, seltener auf Russisch, wobei das Einfügen von anderssprachigen Schimpfwörtern insgesamt zu den häufigsten im Roman realisierten Formen des Sprachwechsels gezählt werden kann, so z.B. „Oh for fuck's sake, wie langweilig sind wir, selbstzufriedene geistig Kranke“ (SŠ: 22). Ganz häufig fallen dem Erzähler populäre Werbeslogans oder irgendwo gehörte bzw. gelesene Zitate ein, die im Erzählfluss meistens in der Originalsprache, also Englisch, rekapituliert werden: „Ich bleibe stehen und kaufe mir in dem Laden an der Ecke einen Kaffee. Feed your caffeine addiction." (SŠ: 13)

Aus Helmichs Perspektive lohnt es sich, bei der Analyse des Sprachwechsels zwischen verschiedenen Arten von Textumgebungen zu unterscheiden und zu prüfen, ob Fremdsprachiges an besonderen Schauplätzen, in bestimmten Szenen oder sonstigen abgrenzbaren Bereichen gehäuft auftritt (Helmich 2016: 30). In diesem Sinne stechen die Romanstellen hervor, in denen es um englischsprachige Posts oder vorformulierte, jedoch nicht realisierte Beiträge im Kopf des Erzählers geht, die sich im ganzen Text verteilen und den unvermittelten Sprachwechsel explizit illustrieren. Die Sprache der sozialen Netzwerke sieht immer schon die Integration englischer Begriffe vor, sodass auch die litauisch-englische Zweisprachigkeit als Ausdruck eines globalen Medialekts gelten kann. Der Bericht über die verbalen und visuellen Posts, Tweets, Chats, etc. wird durch wörtliche Zitate durchdrungen, was zu einer intensiven Sprachmischung führt. Hier ein längeres Beispiel, wie man sie häufig im Roman Stasys Šaltoka finden kann: 
Why 30th bday drives people insane? \#not30yet. Tweet.

Während wir Portobello Burger essen, bekomme ich hunderte Kommentare und Retweets.

What's on ur BEFORE 30th to do list? Tweet.

Noch mehr Kommentare.

Not to die, schreibt jemand. [...]

Wir verabschieden uns, ich komme nach Hause und schlafe ein. Ich erwache, wenn es bereits dunkel ist. Ich sehe, Kanye West hat meinen Tweet beantwortet: TO BECOME A GOD.

Fuckin people. (SŠ: 17)

Wenn der Erzähler an seinem neunundzwanzigsten Geburtstag in der New Yorker Wohnung wach wird, sucht er zunächst nach der besten Perspektive für ein Manhattan-Bild, das sofort in seinem Instagram hochgeladen wird, begleitet von dem englischsprachigen Post: „Klick. \#sublime \#newyorknights \#happydaytome \#blessed \#happiness" (SŠ: 11). Solche Posts vermehren sich auf der Reise durch Asien und helfen dem Erzähler, seine zwischen Begeisterung, Gleichgültigkeit oder Abneigung schwankenden Gemütszustände für sich selbst und für die anderen in wenigen Worten zusammenzufassen.

In den früher erwähnten litauischen Migrationstexten werden die mehrsprachige Kommunikation sowie die transkulturellen Beziehungen häufig auf das Minimum reduziert, wobei als Grund dafür die fehlende Fremdsprachenkompetenz der Migranten gelten soll (Laurušaitè 2019: 46). Im Fall von Stasys Šaltoka ist die oberflächliche, fragmentarische und lakonische Kommunikation nicht durch mangelnde Sprachkenntnisse, sondern durch die Kommunikationsart motiviert. Es handelt sich insofern um eine Art Berufsbzw. Lebensstilidentität konstituierende Authentizität, wenn am Beispiel eines aktiven Nutzers von Social Media gezeigt wird, wie in seinem Bewusstsein verschiedene Sprachen vorhanden sind und abwechselnd zur Artikulation gelangen. Die Bildervielfalt und Sprachmischung dienen bei Grušaitè der Demonstration der Weltläufigkeit, aber auch der Charakterisierung eines sozialen Typus, nämlich eines reiselustigen Digital Native, der die Welt mit Hilfe seines Handys wahrnimmt und präsentiert. Darüber hinaus ist die Zweisprachigkeit ein Charakteristikum, das zur Identitätsbildung bestimmter Zielgruppen beiträgt und globale Community-Zugehörigkeit stärkt.

Stasys Šaltoka reist von New York nach Thailand, von Thailand nach Malaysien, von Malaysien nach Hongkong usw., scheint sich für die fremden Kulturen $\mathrm{zu}$ interessieren, aber klemmt fest an seinem typischen Lifestyle, nämlich Instagram, Luxushotels u.Ä. Die Langeweile und die Abenteuerlust („wir sind hier for some fun“, SŠ: 169) bewegen Stasys und seine Mitreisenden, den ebenfalls aus den USA eingereisten Kenneth Braun und den Neurussen 
Alex Lermontov, dazu, eine Dokumentation über die politische Korruption in Malaysien zu drehen, was zu immer neuen unmittelbaren Begegnungen mit Einheimischen führt. Die Gattung des Reiseberichts impliziert Radaelli zufolge fast immer eine Überschreitung sprachlicher Grenzen und das Betreten anderssprachiger Räume (Radaelli 2011: 76). Auch in Stasys Šaltoka offenbart sich durch Reisebeschreibungen ein mehrsprachiger kultureller und sozialer Raum, der sich mit seiner Vielstimmigkeit den Reisenden aufdrängt. Wenn der Erzähler nach einem 30-stündigen Flug in Bangkok landet, lautet sein erster Eindruck folgendermaßen: „Die Straße voll Hitze und glühender Gesichter, hier ist immer Freitag, hier ist immer zu viel Whisky, zu viele Souvenirs, Schmutz und fremde Sprachen." (SŠ: 58) Obwohl die selbstverliebten Hipster auf ihre eigenen mehrsprachigen Stimmen, sei es auf Instagram oder in der alltäglichen Kommunikation, fixiert bleiben, lässt sich die Vielstimmigkeit der asiatischen Metropolen nicht überhören, wird aber im Roman nur durch den Erzählerbericht, also nicht explizit, wiedergegeben:

Ich gucke beim Vorbeigehen auf all die Muslime und verstehe, wie leicht man Fremdenhass erwecken kann - plötzlich fühle ich mich durch ihre bunte, synthetische und billige Kleidung verärgert, aber auch dadurch, wie sie ihr fettiges Curry mit Händen essen, wie sie ihre verfluchte Barbarensprache sprechen. (SŠ: 164)

An dieser Stelle wird deutlich, wie fremd und undurchdringlich auf den Erzähler die asiatischen Kulturen und Sprachen wirken, was auf eine gewisse Ähnlichkeit zwischen ihm und seinem aus den USA eingereisten Bekannten Kenneth Brown, einem rothaarigen Iren, „geboren an den Ufern der Cardiganbucht“, aufgewachsen in Wales (SŠ: 36), einem Streber nach Ruhm und Anerkennung, hinweist. Kenneth wird vom Erzähler als bornierter Fremdenhasser dargestellt („Diese fuckin piece of shit Muslime...", SŠ: 98), von dem er sich durch seinen spezifischen (post-)sowjetischen Lebenslauf, seinen Weltschmerz und seine existenzphilosophischen Reflexionen zu unterscheiden glaubt. Enttäuscht muss er aber zugeben, dass es gar nicht so viele Unterschiede zwischen ihm und Kenneth gibt: „Vielleicht hatten Kenny und ich vor allem das gemeinsam, dass sowohl Vilnius, die Stadt meiner Kindheit, als auch sein Wales nicht mehr existieren. Im Laufe unseres gar nicht so langen Lebens haben die Strömungen der Geschichte viele Gedächtnisschichten weggeschwemmt, die Kanten entschärft [...]“ (SŠ: 37-38). Stasys erkennt, dass er mit seinem medial vorgespielten Leben dem Waliser viel ähnlicher ist, als er das wahrhaben möchte, so etwa, wenn es um ihre scheinbare Sorge um die Welt, eigentlich aber nur um das eigene Image geht (SŠ: 113). Einen Vorteil glaubt er aber gegenüber Kenneth zu haben, nämlich seine Mehrsprachigkeit, während sich die Sprachkompetenz 
des Walisers auf das Englische sowie seine ganze Weltwahrnehmung auf „das Angelsächsische" beschränkt (SŠ: 133).

Die geteilten Lebenserfahrungen und die Mehrsprachigkeit verbinden Stasys mit dem Russen Alex Lermontov, den er zufällig in Bangkok trifft. Der Name Lermontov kann nicht nur als ironische Anspielung auf die russische Literaturklassik, sondern auch als eine weitere, latente Form literarischer Mehrsprachigkeit gelten. Im Hinblick auf den Sprachwechsel, der durch die Einführung fremdsprachiger Eigennamen geschieht, spricht Radaelli von den Worten, „die stärker als andere mit Bedeutung aufgeladen sind und grundlegende Sinnbeziehungen, ja ganze Vorstellungswelten entstehen lassen“ (Radaelli 2011: 100). Dem Stereotyp eines reichen Oligarchensohns entsprechend, verweist Alex' Familienname zwar auf das in russischer Sprache verfasste Weltliteraturgut (SŠ: 61), aber sein erstsprachlicher Wortschatz beschränkt sich in der aktuellen Kommunikation auf die wenigen Schimpfwörter, wie etwa „Geh nahui“ (SŠ: 93). Die Bedeutung dieser Schimpfwörter ist nur für Alex und Stasys, aber nicht für Kenneth erschließbar und somit können sie als Mittel zur Markierung osteuropäischer Verbundenheit betrachtet werden (auch Stasys selbst reagiert spontan auf einen schlecht schmeckenden Kaffee mit „Nx bl“ (SŠ: 97), was eine Abkürzung vom üblen russischen Schimpfwort „Nahui blyat“ ist und so etwas wie „Verpiss dich“ bedeutet).

Es ist häufig der Fall, dass in den Migrationsromanen, die das Leben litauischer bzw. osteuropäischer (Arbeits-)Migranten schildern, z.B. der Roman Mes vakar buvom saloje (Wir waren gestern auf der Insel, 2011) von Aleksandra Fomina, das Russische als die einzige Verständigungssprache und als Mittel zur Überwindung vom Integrationsschock präsentiert wird. In dem Roman von Grušaitè scheinen die beiden aus dem (post-)sowjetischen Raum stammenden Figuren, Stasys und Alex, sich auf Englisch zu verständigen (SŠ: 63). Dies soll unter anderem als Beweis dafür gelten, wie weit der Transformationsprozess aus einem Osteuropäer in den englischsprachigen Global Player im Fall von Stasys bereits fortgeschritten ist, sodass er für das Denken und Handeln von Alex häufig genauso wenig Verständnis findet wie für die Malaysier oder Thailänder. Das Verhältnis von Stasys zu dem Russen Alex ist insgesamt als ambivalent zu bewerten: Manchmal braucht er ihn als Identifikationsfigur, mit der man gewisse Lebenserfahrungen teilen und in russischer Sprache schimpfen kann, manchmal aber distanziert er sich von Alex, um sich als „richtiger“ New Yorker fühlen zu können (die Kommunikation in englischer Sprache ist offenbar ein Mittel, diese Distanz zu bewahren).

Die Autorin präsentiert einen Litauer, der sich auf seinen Reisen von westlichen fremdenfeindlichen Stereotypen und Klischees leiten lässt oder, was auch ziemlich häufig geschieht, sich die ganze Schuld der „weißen privilegierten 
Männer“ (SŠ: 291) für Diskriminierung, Armut, Hunger usw. auf die eigenen Schultern legt. Die Identifikation mit der „westlichen“ Schuld soll, genauso wie die fortgeschrittene Zweisprachigkeit, die Integration in den westlichen mentalen Raum bestätigen. Grušaitè bringt die Selbstidentifizierung eines Litauers mit der westlichen Welt zum Ausdruck, indem sie seine stereotypische Wahrnehmung des Ostens (Thailand, Malaysien, Hongkong) präsentiert. Der Romanprotagonist kann seine kulturelle Zugehörigkeit bestätigt bekommen, wenn er merkt, dass er den Osten auf eine westliche Art, nämlich als „weißer privilegierter Mann“, konsumieren kann (Repečkaitė 2018). Während er in New York immer noch von den Erinnerungen an seine Jugend in Vilnius heimgesucht wird, personifiziert in der Gestalt einer imaginierten Unbekannten, und eine gewisse Distanz zum amerikanischen oberflächlichen Lifestyle empfindet (SŠ: 19), hört er in Südostasien aufgrund seiner Rasse und unbegrenzten finanziellen Möglichkeiten auf, ein „Osteuropäer“ zu sein. Die Literaturbloggerin Daiva Repečkaitè hat bemerkt, dass die Konsumierung des Ostens auch durch den unterschiedlichen Umgang der Autorin mit verschiedenen, im Roman explizit oder latent vorkommenden Fremdsprachen geschieht: So werden z.B. die englischen Eigennamen konsequent ins Litauische transkribiert („Džanet“; “Izabelë“, etc.), die südostasiatischen Toponyme dagegen stehen meistens in englischer Sprache, als ob Grušaitè nicht wüsste, wie man sie aussprechen soll (Repečkaitè 2018). Ähnliches gilt auch für asiatische Eigennamen, die sogar durch kreative Alternativen ersetzt werden, so etwa der Name eines Zimmermädchens: „wir nennen sie Mel, weil wir ihren Namen nicht aussprechen können“ (SŠ: 279). Aus imagologischer Sicht ist immer die Frage, ob die Stereotypisierungen auf der Textebene dekonstruiert oder im Gegenteil verstärkt werden, was im Fall des Romans Stasys Šaltoka im Zusammenhang mit seiner Mehrsprachigkeit diskutiert werden kann. Die sarkastischen Bemerkungen über den westlichen Konsum des Ostens, z.B. die Fremde sei nur als „exotische Lokation“ für Instagram-Bilder interessant (SŠ: 107), sollen die im Roman enthaltene Kritik an so einer begrenzten Perspektive betonen; die weitgehend zum Schweigen gebrachte Sprachen- bzw. Varietäten-Vielfalt und die Reduzierung der Kommunikation auf Englisch als globale Verständigungssprache zeugen jedoch von sprachlicher und kultureller Hegemonie, wie sie auch aus der Kolonialzeit für die Region bekannt ist, und somit von gewisser Inkonsequenz des auf Überwindung von Stereotypen aufbauenden Romankonzepts. 


\section{Schlusswort}

Der gegen konventionellen Sprachpurismus gerichtete kreative Sprachgebrauch belegt, dass die junge litauische Autorengeneration gerne Sprach- und Kulturgrenzen bricht. Die Autorinnen Unè Kaunaitè, Gabija Grušaitè u.a. zeigen, was das literarische Spiel mit der Mehrsprachigkeit vermag, nämlich subversiv sein, Komik erzeugen, zugleich aber auf Probleme sprachlicher Dominanz und somit der politischen, sozialen und kulturellen Diskriminierung verweisen, Gruppenzugehörigkeit ausdrücken, etc. Wie die litauische Literatur mit Mehrsprachigkeit umgeht, diese explizit oder implizit in den Texten klingen lässt, ist auch ein Zeichen dafür, inwieweit sich bestimmte Texte als transkulturell bezeichnen lassen und den aktuellen Ton der europäischen bzw. globalen Migrations- und Mobilitätsliteratur treffen.

Der Roman Stasys Šaltoka von Gabija Grušaitè verdeutlicht einen bewussten Versuch, sich von den Zwängen der Provinzialität zu befreien, was sowohl auf der Handlungs- als auch auf der Ausdrucksebene zu beobachten ist. Auf der Handlungsebene fallen der ironische Umgang der Autorin mit dem neuen sozialen Typus der Instagram-Generation, die geographisch besonders breite Gestaltung des Handlungsraums und das für die litauische Literatur untypische Interesse an globalen Themen, so etwa die politische Korruption in Malaysien, das Hochwasser in Bangkok, etc. auf. Stilistisch zeichnet sich der Roman durch seinen umgangssprachlichen Ton, vor allem aber durch seine explizite Zweisprachigkeit aus, die möglichst authentisch die Stimme der eigenen Generation illustrieren soll. Für die medienbesessenen Global Player stellt Englisch kein Kommunikationshindernis dar, im Unterschied etwa zu den litauischen bzw. osteuropäischen (Arbeits-)Migranten, deren unzureichende Fremdsprachenkompetenz häufig als Ursache für Missverständnisse gilt. Ganz im Gegenteil scheint der Romanprotagonist sich mittels des Englischen nicht nur mit seinen amerikanischen Freunden, sondern auch mit allen auf Reisen getroffenen Menschen, seien sie aus der Mongolei oder aus Thailand, verständigen zu können. In diesem Sinne inszeniert die Autorin eine Art Auseinandersetzung mit der „überlegenen“ Sprache, die einerseits kulturelle Polyphonie verstummen lässt, andererseits aber die bereits vollzogene Integration des Protagonisten in die westliche Kulturwelt beweisen soll.

Solche Texte wie Stasys Šaltoka setzen auch mehrsprachige Leser voraus, denen das Verständnis fremder Sprach- und Kulturelemente zugetraut werden kann und die in der Lage sind, anderssprachige Passagen als spezifischen Soziolekt oder mediale Anspielung zu interpretieren. Der literarische Sprachwechsel erscheint in diesem Zusammenhang nicht mehr als Normbruch, als Verrat an der Erstsprache, auch nicht mehr als Quelle der Misskommunikation, sondern trägt zur Realisierung der Absicht der Autorin 
Sprachwechsel in der neuesten litauischen Migrations- und Mobilitätsliteratur

bei, „eine Art anthropologischen Text“ zu verfassen, der sich nicht nur als transsprachlich, sondern auch als transkulturell und transmedial bezeichnen lässt.

\author{
Rūta Eidukevičienè \\ ruta.eidukeviciene@vdu.lt \\ Vytautas Magnus University \\ LITHUANIA
}

\title{
Literaturverzeichnis
}

Dagnino, A. 2015. Transcultural Writers and Novels in the Age of Global Mobility. West Lafayette: Purdue University Press.

Dembeck, T. 2020. Basisverfahren literarischer Mehrsprachigkeit. - T. Dembeck, R. Parr, Hrsg., Literatur und Mehrsprachigkeit. Ein Handbuch. Tübingen: Narr/ Francke/Attempto, 123-166.

Dembeck, T., Uhrmacher, A. 2016. Vorwort: Erfahren oder erzeugt? Zum literarischen Leben der Sprachdifferenz. - T. Dembeck, Hrsg., Das literarische Leben der Mehrsprachigkeit. Methodische Erkundungen. Heidelberg: Universitätsverlag C. Winter, 9-18.

Eurostat. EU citizens living in another Member State - statistical overview 17/07/ 2020. - https://ec.europa.eu/eurostat/statistics-explained/pdfscache/68490.pdf (20.07.2020).

Gimbutaitė, M. 2017. Kaip kūrybos kalbą renkasi užsienyje gyvenantys lietuvių autoriai? - https://www.15min.lt/kultura/naujiena/literatura/kaip-kurybos-kalbarenkasi-uzsienyje-gyvenantys-lietuviu-autoriai-286-759498 (20.07.2020).

Grušaitè, G. 2018. Stasys Šaltoka. Vilnius: Lapas.

Helmich, W. 2016. Ästhetik der Mehrsprachigkeit. Zum Sprachwechsel in der neueren romanischen und deutschen Literatur. Heidelberg: Universitätsverlag C. Winter.

Kačkutè, E. 2013. Apie kutūrinị (ne)priklausymą. - https://www.bernardinai.lt/201312-28-egle-kackute-apie-kulturini-ne-priklausyma/ (20.07.2020).

Kačkutè, E. 2018. Reflections on Belonging. Contemporary Lithuanian Women's Writing on Mobility and Migration: Staponkutè, Pukytė, Žagrakalytė. - http:// vilniusreview.com/nonfiction/reflections-on-belonging/282-contemporary-lithuanian-women-s-writing-on-mobility-and-migration-staponkute-pukyte-zagrakalyte (20.07.2020).

Kaniavienè, D. 2018. Šaltoka \#taipkaipšiandien. - https://literaturairmenas.lt/literatura/dainora-kaniaviene-saltoka-taipkaipsiandien (20.07.2020).

Laurušaitė, L. 2019. Kalbos transformacinè galia lietuvių ir latvių (e)migracijos prozoje. - Lituanistica, 62, 39-52.

Radaelli, G. 2011. Literarische Mehrsprachigkeit. Sprachwechsel bei Elias Canetti und Ingeborg Bachmann. Berlin: Akademie Verlag. 
EIDUKEVIČIENĖ

Repečkaitè, D. 2018. Rezension zu Gabija Grušaitė, „Stasys Šaltoka: vieneri metai“. https://www.daivarepeckaite.com/2018/01/gabija-grusaite-stasys-saltoka/ (20.07.2020).

Satkauskytė, D. 2011. Egzilinė (ne)tapatybè naujausioje lietuvių emigrantų literatūroje. - Oikos, 2, 120-129.

Schmitz-Emans, M. 2004. Literatur und Vielsprachigkeit: Aspekte, Themen, Voraussetzungen. - M. Schmitz-Emans, Hrsg., Literatur und Vielsprachigkeit. Heidelberg: Synchron, 11-26.

Sturm-Trigonakis, E. 2007. Global Playing in der Literatur. Ein Versuch über die Neue Weltliteratur. Würzburg: Königshausen u. Neumann.

Vyšniauskas, K. 2018. Ką daryti, kai tau 30, turi butą prestižiniame rajone, kalną instagramo sekejjų, bet viduje - tuščia: Pokalbis su rašytoja Gabija Grušaite. - https:// www.moteris.lt/lt/veidai/g-28095-ka-daryti-kai-tau-30-turi-buta-prestiziniamerajone-kalna-instagramo-sekeju-bet-viduje-tuscia (20.07.2020). 